OPEN ACCESS

Edited by:

Ulrike Stein,

Charité - Universitätsmedizin Berlin,

Germany

Reviewed by:

Mathias Dahlmann,

Charité - Universitätsmedizin Berlin,

Germany

Hui Guo,

First Affiliated Hospital of Xi'an

Jiaotong University, China

*Correspondence:

Chunyan Zeng

zcy896@163.com

Youxiang Chen

chenyx102@126.com

${ }^{t}$ These authors have contributed equally to this work

Specialty section: This article was submitted to Gastrointestinal Cancers, a section of the journal

Frontiers in Oncology

Received: 11 August 2020 Accepted: 27 January 2021

Published: 05 March 2021

Citation:

Liu B-X, Tang C-T, Dai X-J, Zeng L, Cheng F, Chen $Y$ and Zeng $C$ (2021)

Prognostic Value of S100P Expression

in Patients With Digestive System Cancers: A Meta-Analysis.

Front. Oncol. 11:593728.

doi: 10.3389/fonc. 2021.593728

\section{Prognostic Value of S100P Expression in Patients With Digestive System Cancers: A Meta-Analysis}

\author{
Bi-Xia Liu ${ }^{1 \dagger}$, Chao-Tao Tang ${ }^{1 \dagger}$, Xi-Jian Dai ${ }^{2,3}$, Ling Zeng ${ }^{1}$, Fei Cheng ${ }^{1}$, Youxiang Chen ${ }^{1 *}$ \\ and Chunyan Zeng ${ }^{1 *}$
}

\begin{abstract}
${ }^{1}$ Department of Gastroenterology, The First Affiliated Hospital of Nanchang University, Nanchang, China, ${ }^{2}$ Department of Psychiatry, Faculty of Medicine, Chinese University of Hong Kong, Hong Kong, China, ${ }^{3}$ Shenzhen Mental Health Centre, Shenzhen Kangning Hospital, Shenzhen, China
\end{abstract}

Background: Digestive system cancers (DSCs) are associated with high morbidity and mortality. S100P has been reported as a prognostic biomarker in DSCs, but its prognostic value remains controversial. Accordingly, we conducted a meta-analysis to investigate whether S100P is correlated with overall survival (OS) of patients with DSCs. The relationship between S100P and clinicopathological features was also evaluated.

Methods: We systematically searched PubMed, Embase, Web of Science and Cochrane Library for eligible studies up to January 2020. In total, 16 publications with 1,925 patients were included.

Results: S100P overexpression was associated with poor OS of patient with DSCs $(\mathrm{HR}=1.54,95 \% \mathrm{Cl}: 1.14-2.08, \mathrm{P}=0.005)$. When stratified by anatomic structure, S100P overexpression was associated with poor prognosis in non-gastrointestinal tract cancers $(\mathrm{HR}=1.98,95 \% \mathrm{Cl}: 1.44-2.72, \mathrm{P}<0.001)$ but not in gastrointestinal tract cancers $(\mathrm{HR}=1.09,95 \% \mathrm{Cl}: 0.66-1.81, \mathrm{P}=0.727)$. When stratified by tumor type, S100P overexpression predicted poor $\mathrm{OS}$ in cholangiocarcinoma $(\mathrm{HR}=2.14,95 \% \mathrm{Cl}: 1.30$ 3.50, $\mathrm{P}=0.003)$ and hepatocellular carcinoma ( $\mathrm{HR}=1.91,95 \% \mathrm{Cl}: 1.22-2.99, \mathrm{P}=0.005)$ but not in gastric cancer $(\mathrm{HR}=0.97,95 \% \mathrm{Cl}: 0.65-1.45, \mathrm{P}=0.872)$, colorectal cancer $(\mathrm{HR}=1.18,95 \% \mathrm{Cl}: 0.32-4.41, \mathrm{P}=0.807)$, gallbladder cancer $(\mathrm{HR}=1.40,95 \% \mathrm{Cl}: 0.84-$ 2.34, $P=0.198)$, and pancreatic cancer ( $\mathrm{HR}=1.92,95 \% \mathrm{Cl}$ : 0.99-3.72, $\mathrm{P}=0.053)$. Furthermore, high S100P expression was significantly associated with distant metastasis $(\mathrm{OR}=3.58, \mathrm{P}=0.044)$, advanced clinical stage $(\mathrm{OR}=2.03, \mathrm{P}=0.041)$ and recurrence $(\mathrm{OR}=1.66, \mathrm{P}=0.007)$.

Conclusion: S100P might act as a prognostic indicator of non-gastrointestinal tract cancers.

Keywords: S100 calcium-binding protein P (S100P), prognostic, meta-analysis, digestive system, cancers 


\section{INTRODUCTION}

Digestive system cancers (DSCs) are associated with high morbidity and mortality, which mainly consist of gastric cancer, colorectal cancer, hepatocellular carcinoma, esophageal cancer, pancreatic cancer, cholangiocarcinoma, and gallbladder cancer. Although some advances have been made in diagnosis and treatment in recent years, DSCs remain major threats for human health due to the high morbidity and mortality rates worldwide (1). Although numerous biomarkers involved in DSCs have been identified, sensitive imaging methods and biomarkers are scarce, and many DSC patients are identified at an advanced clinical stage, resulting in poor prognosis due to the high incidence of lymph node invasion, distant metastasis and local recurrence $(2,3)$. Moreover, patients with the same tumor characteristics, such as tumor size, tumor differentiation and clinical stage, may suffer from diverse clinical outcomes (4). Therefore, reliable new biomarkers are needed.

S100P is a member of the large family of S100 calcium-binding proteins, and it was initially isolated from human placenta $(5,6)$. S100P regulates a number of cellular processes through multiple signal pathways (7-11), and it is widely expressed in both normal and malignant tissues. In normal adult tissues, S100P exhibits the highest expression in the placenta and stomach (12). Overexpression of $\mathrm{S} 100 \mathrm{P}$ has been detected in several tumors, including cholangiocarcinoma (11), colorectal cancer (13-15), lung cancer (16), pancreatic cancer (17), breast cancer (18), ovarian cancer (19), gastric cancer (20), and prostate carcinoma (12). S100P interacts with a number of signaling molecules both extracellularly and intracellularly $(21,22)$, and it has been demonstrated to mediate tumor growth, drug resistance, invasion, and metastasis $(8,13,16,17$, $23-25)$. S100P is a potential biomarker for diagnosis and a potential target molecule for therapeutic intervention $(8,26,27)$. Therefore, our primary hypothesis is that overexpression of $\mathrm{S100P}$ is associated with a poor prognosis in DSCs. The objective of the present metaanalysis study was to utilize existing literature to evaluate the prognostic value of S100P in DSCs.

\section{METHODS}

\section{Data Sources and Search Strategy}

The PRISMA statement was followed in the systematic review and meta-analysis (28). We searched PubMed, Embase, Web of Science, and Cochrane Library up to January 2020. Studies that assessed the association between $\mathrm{S} 100 \mathrm{P}$ expression and the survival outcome of DSCs patients were included in the metaanalysis. References reported in the included studies were also manually reviewed to identify potential missing studies in the initial search. The search terms were as follows: (S100P, S100 calcium-binding protein P, MIG9, or migration-inducing gene 9 protein) and (cancer, tumor, carcinoma, neoplasm, or malignancy) and (prognosis, prognostic, survival, outcome, or metastasis). No language restrictions were applied. Two investigators (Bi-Xia Liu and Chao-Tao Tang) searched the databases independently.

\section{Selection Criteria}

Studies were eligible for inclusion based on the following criteria: (1) investigation of the relationship between S100P expression and overall survival (OS) or disease-free survival (DFS) in DSC patients; (2) data reported for hazard ratio (HR) and 95\% confidence interval (95\% CI) directly or indirectly (data allowing calculation of these values); (3) S100P expression was detected in the tumor tissues of DSC patients; (4) patients were divided into high and low expression subgroups according to the cut-off value of S100P; (5) published in full text; and (6) human studies. The exclusion criteria were as follows: (i) insufficient data; (ii) previously published or smaller sample size studies were excluded after identification of duplicate publications or duplicate data; and (iii) reviews, letters, case reports, conference abstracts, or laboratory studies. Ethical approval and patient consent were not required for this meta-analysis.

\section{Data Extraction and Quality Assessment}

Two investigators (Bi-Xia Liu and Chao-Tao Tang) independently evaluated and extracted all the essential data from enrolled articles. Any disagreements were resolved through discussion. The following information was extracted from the included studies: first author's name; publication year; number of cases; gender; age; country; cancer type; test method; cut-off value for high expression of S100P; outcome; HR and corresponding 95\% CI for OS and DFS; and clinicopathological characteristics, including differentiation, lymph node metastasis, distant metastasis, $\mathrm{T}$ stage, clinical stage, vascular invasion, and recurrence. The quality of the included studies was assessed according to the Newcastle-Ottawa Scale (NOS) (29). The NOS contains eight items and categorized into three dimensions as follows: selection ( $0-4$ points), comparability ( $0-2$ points) and outcome assessment ( $0-3$ points). The NOS score ranges from 0 to 9 , and studies with NOS scores $\geq 6$ are considered high quality.

\section{Statistical Analysis}

Statistical analyses were conducted with Stata MP16 Software (STATA Corp., College Station, Texas, USA). The HRs and 95\% CIs were utilized to assess the prognostic value of S100P expression on the survival of patients with DSCs. HR $>1$ with $\mathrm{p}$-value $<0.05$ indicated a poor prognosis of S100P overexpression in DSC patients. The odds ratios (ORs) with corresponding $95 \%$ CIs were used to analyze the association between the S100P expression level and the clinicopathological parameters. The HRs and 95\% CIs were obtained directly from the published studies or extracted from the Kaplan-Meier survival curves by Engauge Digitizer version 12.1. Heterogeneity among the enrolled studies was quantitated with $\mathrm{I}^{2}$ statistics. The random-effect model was applied if heterogeneity was present $\left(\mathrm{I}^{2} \geq 50 \%\right.$ or $\left.\mathrm{P} \leq 0.1\right)$, and if heterogeneity was not present, the fixed-effect model was used. Subgroup analysis, as stratified by anatomic structure, cancer type, sample size, and study region, was performed. Sensitivity analysis was conducted to assess the reliability of the results. Metaaggression analysis was conducted to identify the source of heterogeneity. Publication bias was measured by Begg's test and Egger's test. P-value $<0.05$ was considered statistically significant. 


\section{RESULTS}

\section{Study Selection}

The literature search and selection process are shown in Figure 1. In total, 1,863 articles were retrieved in the initial search. After removing the duplicate articles, 1,194 articles were entered into further screening. In total, 1,155 articles were discarded after careful review of the title and abstract. Subsequently, the full texts of the remaining 39 articles were evaluated, and 23 articles were excluded due to the following reasons: insufficient data in six articles; S100P not the main focus in 15 articles; unavailable fulltext in one article; and overlapped full-text in one article. Finally, the remaining 16 articles $(11,13,20,23,27,30-40)$, comprising 1,925 patients, were enrolled in the current meta-analysis.

\section{Characteristics of Included Studies}

The basic characteristics of the included studies are shown in Table 1. The included studies were published between 2009 and 2019. Specifically, one study by Aishima et al. investigated two cancer subtypes and was marked as Aishima et al. (1) and Aishima et al. (2) (38). Therefore, 16 articles with 17 studies were included. Among the included articles, one was published in Chinese, and the others were published in English. The patients were enrolled from the following five countries: China, Japan, Thailand, Portugal, and Italy. Four studies reported on patients with gastric cancer (GC) $(20,30,39,40)$, and three studies focused on patients with colon cancer (CC) or colorectal cancer (CRC) $(13,23,27)$. Two studies reported on patients with cholangiocarcinoma (CCA) $(11,34)$, and four studies reported

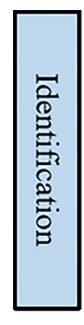

\begin{tabular}{|c|c|}
\hline $\begin{array}{c}\text { Records identified through } \\
\text { database searching } \\
(\mathrm{n}=1863)\end{array}$ & $\begin{array}{c}\text { Additional records identified } \\
\text { through other sources } \\
(\mathrm{n}=0)\end{array}$ \\
\hline
\end{tabular}

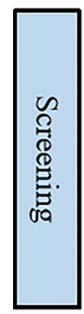

Records after duplicates removed
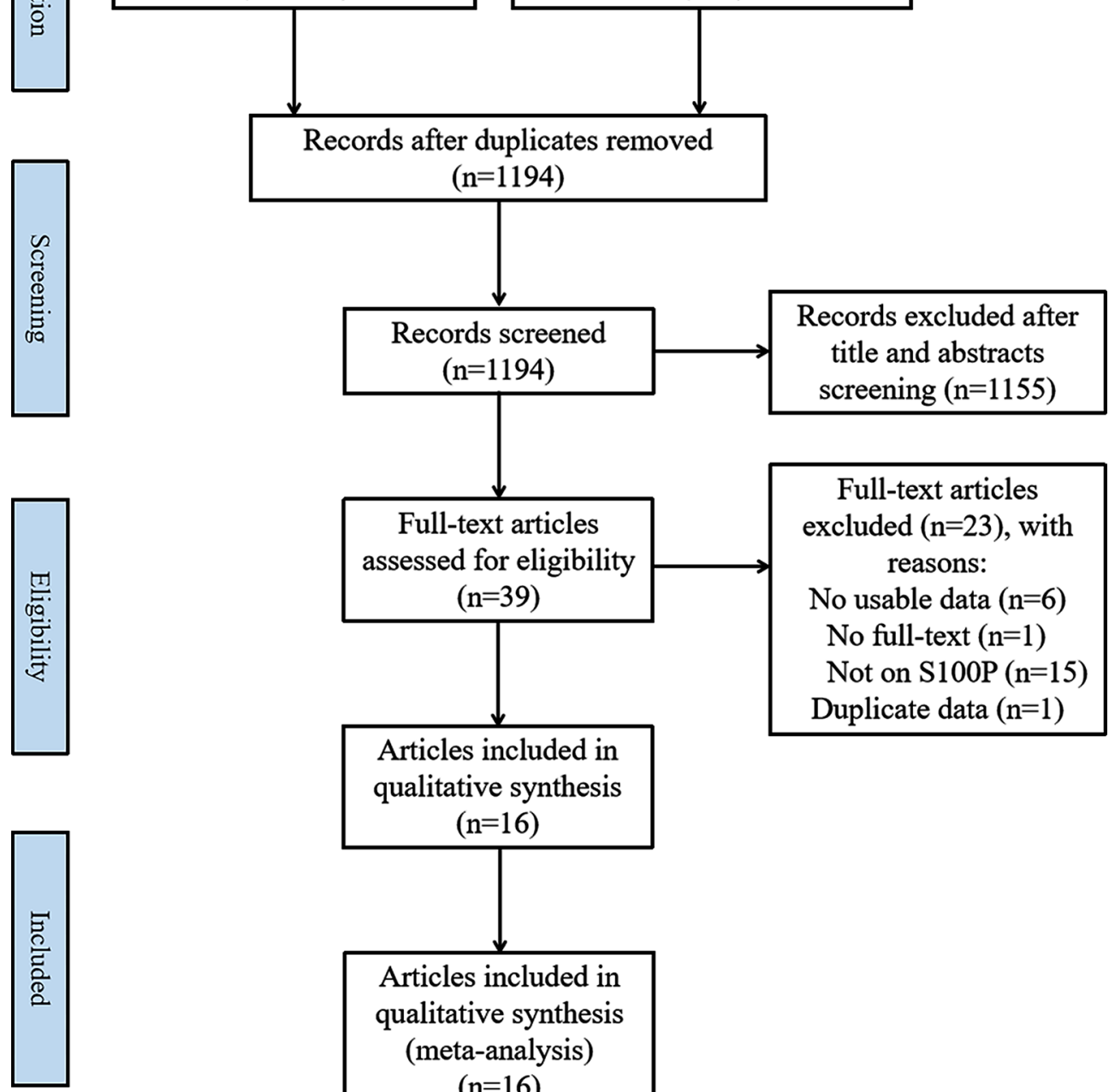

Articles included in qualitative synthesis $(\mathrm{n}=16)$

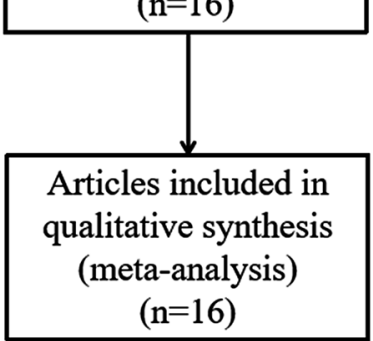

FIGURE 1 | Flow diagram of the search and selection process. 
on patients with intrahepatic cholangiocarcinoma (ICC) $(32,37$, 38). One study reported on patients with extrahepatic cholangiocarcinoma (ECC) or extrahepatic bile duct carcinoma (EHBDC) (36), and one study reported on patients with hepatocellular carcinoma (HCC) (35). One study reported on patients with gallbladder cancer (GBC) (33), and one study reported on patients with pancreatic cancer (PC) (31). Because CCA originates from the epithelium of the bile duct and is further classified into ICC and ECC (41), the CCA, ICC, and EHBDC were categorized into the same group in the following subgroup analysis. S100P expression in tumor tissues was detected by IHC in 15 studies, RT-PCR in one study and western blotting (WB) in one study.

The NOS scores of the included studies ranged from 6 to 8 , indicating high-quality of the included studies (Table 2).

\section{Correlation of High S100P Expression With OS in Digestive System Cancers}

Data for the association between S100P expression and OS were extracted from all the included 17 studies $(11,13,20,23,27,30-$ $40)$. The heterogeneity was significant among these studies $\left(\mathrm{I}^{2}=\right.$ $78.0 \%, \mathrm{P}<0.001$ ); therefore, the random-effects model was used. The pooled result revealed that high S100P expression was significantly associated with poor OS of DSC patients $(n=17$, pooled $\mathrm{HR}=1.54,95 \% \mathrm{CI}=1.14-2.08, \mathrm{P}=0.005$ ) (Table 3 and Figure 2). In addition, subgroup analysis was performed for further investigation. When the cancers were stratified by anatomic structure, the data showed that high S100P expression remained a significant factor of poor OS in patients with non-gastrointestinal tract cancer $(\mathrm{n}=10, \mathrm{HR}=1.98,95 \% \mathrm{CI}=$ 1.44-2.72, $\mathrm{P}<0.001)$ but not in patients with gastrointestinal tract cancer $(n=7, H R=1.09,95 \% C I=0.66-1.81, P=0.727$; Table 3 and Figure 3A). When the subgroup analysis was conducted by cancer type, the data revealed that the high S100P level significantly led to poor OS in patients with CCA $(n=7$, $\mathrm{HR}=2.14,95 \% \mathrm{CI}=1.30-3.50, \mathrm{P}=0.003)$ and in patients with HCC $(\mathrm{n}=1, \mathrm{HR}=1.91,95 \% \mathrm{CI}=1.22-2.99, \mathrm{P}=0.005)$ but not in patients with $\mathrm{GC}(\mathrm{n}=4, \mathrm{HR}=0.97,95 \% \mathrm{CI}=0.65-1.45, \mathrm{P}=0.872)$, CRC $(\mathrm{n}=3, \mathrm{HR}=1.18,95 \% \mathrm{CI}=0.32-4.41, \mathrm{P}=0.807)$, GBC $(\mathrm{n}=1$, $\mathrm{HR}=1.40,95 \% \mathrm{CI}=0.84,2.34, \mathrm{P}=0.198)$ and $\mathrm{PC}(\mathrm{n}=1, \mathrm{HR}=1.92$, 95\% CI=0.99-3.72, $\mathrm{P}=0.053$; Table 3 and Figure 3B). Furthermore, S100P expression was also a prognostic factor of poor OS in studies with large sample size $(n=6, H R=1.57,95 \%$ $\mathrm{CI}=1.06-2.33, \mathrm{P}=0.024)$ and in an Asian population $(\mathrm{n}=15$, $\mathrm{HR}=1.57,95 \% \mathrm{CI}=1.10-2.26, \mathrm{P}=0.014)$ but not in studies with small sample size $(\mathrm{n}=11, \mathrm{HR}=1.50,95 \% \mathrm{CI}=0.93-2.43, \mathrm{P}=0.098)$ and in an European population $(\mathrm{n}=2, \mathrm{HR}=1.31,95 \% \mathrm{CI}=0.87-$ 1.98, $\mathrm{P}=0.190$; Table 3 and Figures 3C, D). As the detect methods and the cut-off value of S100P in studies included in meta-analysis were not consistent, we further performed subgroup analysis according to detect methods and cut-off value. The results showed that $\$ 100 \mathrm{P}$ expression was a prognostic factor of poor OS in studies when S100P detected by IHC $(\mathrm{n}=15, \mathrm{HR}=1.57,95 \% \mathrm{CI}=1.13-2.18, \mathrm{P}=0.007)$ and detected by RT-PCR $(n=1, H R=1.80,95 \% C I=1.02-3.19$, $\mathrm{P}=0.044)$ but not in studies when S100P detected by WB $(n=1$,
$\mathrm{HR}=0.55,95 \% \mathrm{CI}=0.13-2.30, \mathrm{P}=0.413$; Table 3 and Supplementary Figure 1). Furthermore, S100P expression was also a prognostic factor of poor OS in studies when the cut-off values were stained grade $2+(\mathrm{n}=5, \mathrm{HR}=2.04,95 \% \mathrm{CI}=1.06-3.93$, $\mathrm{P}=0.032)$ and stained cells $1 \%(\mathrm{n}=3, \mathrm{HR}=1.84,95 \% \mathrm{CI}=1.27-$ 2.67, $\mathrm{P}=0.001$; Table 3 and Supplementary Figure 2). The DFS was not analyzed because only one study was eligible.

\section{Correlation of S100P and Clinicopathological Characteristics}

The relationships between S100P expression and clinicopathological features are illustrated in Table 4. No significant heterogeneity was observed among studies regarding differentiation grade $\left(\mathrm{I}^{2}=0\right.$, $\mathrm{P}=0.683)$, $\mathrm{T}$ stage $\left(\mathrm{I}^{2}=0, \mathrm{P}=0.399\right)$, vascular invasion $\left(\mathrm{I}^{2}=44.8\right.$, $\mathrm{P}=0.163)$ and recurrence $\left(\mathrm{I}^{2}=48.3, \mathrm{P}=0.144\right)$; therefore, the fixedeffects model was applied. Nevertheless, significant heterogeneity was detected among studies regarding gender $\left(\mathrm{I}^{2}=51.4, \mathrm{P}=0.036\right)$, lymph node metastasis $\left(\mathrm{I}^{2}=69.6, \mathrm{P}=0.001\right)$, distant metastasis $\left(\mathrm{I}^{2}=81.3\right.$, $\mathrm{P}=0.001)$ and clinical stage $\left(\mathrm{I}^{2}=77.0, \mathrm{P}=0.001\right)$; thus, the random effects model was used. We observed that high S100P expression was significantly correlated with certain phenotypes of tumor aggressiveness, such as distant metastasis ( $\mathrm{OR}=3.58$, 95\% CI: $1.04-$ 12.36, $\mathrm{P}=0.044)$, advanced clinical stage $(\mathrm{OR}=2.03$; 95\% $\mathrm{CI}=1.03$ $4.01 ; \mathrm{P}=0.041)$ and recurrence $(\mathrm{OR}=1.66 ; 95 \% \mathrm{CI}=1.15-2.38$; $\mathrm{P}=0.007)$. This finding indicated that S100P may promote tumor invasion and recurrence. However, no significance correlation was found between S100P expression and gender $(\mathrm{OR}=0.91,95 \% \mathrm{CI}$ : $0.63-1.32, \mathrm{P}=0.617)$, differentiation grade $(\mathrm{OR}=1.09,95 \% \mathrm{CI}: 0.75-$ $1.57, \mathrm{P}=0.652)$, lymph node metastasis ( $\mathrm{OR}=1.66,95 \% \mathrm{CI}: 0.93-2.97$, $\mathrm{P}=0.084)$, $\mathrm{T}$ stage $(\mathrm{OR}=0.89,95 \% \mathrm{CI}: 0.59-1.36, \mathrm{P}=0.598)$, or vascular invasion ( $\mathrm{OR}=0.99,95 \% \mathrm{CI}$ : $0.63-1.57, \mathrm{P}=0.978)$.

We also analyzed the correlation between S100P expression and the clinicopathological factors in GC, CRC, and CCA. HCC, GBC, and PC were not analyzed because only one study was included for the three cancers. The results of pooled OR and 95\% CI showed that there was significant correlation between S100P expression and gender in $\mathrm{GC}(\mathrm{P}=0.019)$ but that there was no significant correlation between $\mathrm{S} 100 \mathrm{P}$ expression and lymph node metastasis $(\mathrm{P}=0.556)$. In addition, there was no significant association between S100P expression and gender $(\mathrm{P}=0.349)$, differentiation grade $(\mathrm{P}=0.926)$, lymph node metastasis $(\mathrm{P}=0.113)$ or clinical stage $(\mathrm{P}=0.274)$ in patients with $\mathrm{CRC}$. For patients with CCA, there was significant correlation between S100P expression and lymph node metastasis $(\mathrm{P}<0.001)$, but there was no significant correlation between S100P expression and gender $(\mathrm{P}=0.671)$ or differentiation grade $(\mathrm{P}=0.105)$ (Supplementary Materials: Table S1).

\section{Sensitivity Analysis and Meta-Regression Analysis}

The stability and reliability of pooled HRs for OS were evaluated by sensitivity analysis. The results demonstrated that the conclusions were stable and reliable because the pooled HRs were not significantly affected by any individual study (Figure 4). Metaregression analysis showed that the anatomic structure $(\mathrm{P}=0.758)$, cancer type $(\mathrm{P}=0.925)$, sample size $(\mathrm{P}=0.456)$, study region 
TABLE 1 | Baseline characteristics of studies included in the meta-analysis.

\begin{tabular}{|c|c|c|c|c|c|c|c|c|c|c|c|c|}
\hline Author & Year & Country & $\begin{array}{l}\text { Cancer } \\
\text { type }\end{array}$ & Case number/male (n) & $\begin{array}{c}\text { Age (years) } \\
\text { Median/mean } \\
\text { (range) }\end{array}$ & $\begin{array}{l}\text { Test } \\
\text { method }\end{array}$ & $\begin{array}{l}\text { cut-off of high } \\
\text { expression }\end{array}$ & $\begin{array}{l}\text { High S100P } \\
\text { expression: } \\
\text { n (\%) }\end{array}$ & Outcome & $\begin{array}{c}\text { HR }(95 \% \mathrm{Cl}) \text { for } \\
\text { OS }\end{array}$ & $\begin{array}{c}\text { HR } \\
\text { availability }\end{array}$ & $\begin{array}{l}\text { NOS } \\
\text { score }\end{array}$ \\
\hline Carneiro et al. (30) & 2019 & Portugal & GC & $318 / 181$ & Range:32-95 & $\mathrm{IHC}$ & stained graded:3+ & $231(72.6 \%)$ & OS & $1.09(0.78-1.52)$ & Indirectly & 8 \\
\hline Ge et al. (20) & 2013 & China & GC & 156/NR & $N R$ & RT-PCR & Median value & $82(52.6 \%)$ & OS & $1.80(1.12-3.51)$ & Indirectly & 6 \\
\hline Zhao et al. (39) & 2010 & China & GC & $121 / 86$ & $\begin{array}{l}\text { Mean:58 } \\
\text { Range:26-82 }\end{array}$ & $\mathrm{IHC}$ & $\begin{array}{l}\text { stained graded } \geq 2 \\
+\end{array}$ & $64(52.9 \%)$ & OS & $0.77(0.47-1.26)$ & Indirectly & 6 \\
\hline Jia et al. (40) & 2009 & China & GC & 93/NR & NR & $\mathrm{IHC}$ & stained cells > 5\% & $44(47.3 \%)$ & OS & $0.62(0.38-0.93)$ & Indirectly & 6 \\
\hline Shen et al. (23) & 2016 & China & $\mathrm{CC}$ & $125 / 49$ & $\begin{array}{l}\leq 60: 71 \\
>60: 54\end{array}$ & $\mathrm{HC}$ & stained graded $\geq 2+$ & $55(44 \%)$ & OS & $3.33(2.11-5.26)$ & Directly & 7 \\
\hline Dong et al. (13) & 2014 & China & $\mathrm{CRC}$ & $91 / 39$ & $\begin{array}{l}\text { Mean:60.09 } \\
\text { Range:34-81 }\end{array}$ & $\mathrm{HC}$ & H score $\geq 102$ & $34(37.4 \%)$ & OS & $0.66(0.25-1.74)$ & Indirectly & 7 \\
\hline Wang et al. (27) & 2012 & China & $\mathrm{CRC}$ & $96 / 61$ & $\begin{array}{l}\text { Mean:62.4 } \\
\text { range: } 28-92\end{array}$ & WB & NR & 63(65.6\%) & OS & $0.55(0.13-2.28)$ & Indirectly & 7 \\
\hline Wu et al. (11) & 2016 & Thailand & CCA & $78 / 50$ & $\begin{array}{l}<60: 50 \\
\geq 60: 28\end{array}$ & $\mathrm{HC}$ & stained graded $\geq 3+$ & $46(59 \%)$ & OS & $3.37(1.60-7.12)$ & Indirectly & 6 \\
\hline Sato et al. (34) & 2013 & Japan & CCA & $33 / 21$ & Mean:73 & $\mathrm{IHC}$ & stained graded $\geq 1+$ & $29(88 \%)$ & OS & $0.45(0.13-1.56)$ & Indirectly & 6 \\
\hline $\begin{array}{l}\text { Sarcognato et al. } \\
\text { (32) }\end{array}$ & 2019 & Italy & ICC & $61 / 26$ & $\begin{array}{l}\text { Mean:67 } \\
\text { Range:35-82 }\end{array}$ & $\mathrm{HC}$ & $\begin{array}{l}\text { stained graded as } \\
2+\end{array}$ & 12(19.7\%) & OS & $1.66(1.08-2.55)$ & Directly & 6 \\
\hline Tsai et al. (37) & 2012 & China & ICC & $112 / 60$ & $\begin{array}{l}\text { Mean:60.8 } \\
\text { Range:30-87 }\end{array}$ & $\mathrm{HC}$ & stained cells > $1 \%$ & $59(52.7 \%)$ & OS & $1.62(1.14-2.30)$ & Indirectly & 6 \\
\hline $\begin{array}{l}\text { Aishima et al. (1) } \\
\text { (38) }\end{array}$ & 2011 & Japan & Perihilar ICC & $41 / \mathrm{NR}$ & $N R$ & $\mathrm{HC}$ & stained graded $\geq 2+$ & 28(68.3\%) & OS & $1.71(0.65-4.51)$ & Indirectly & 6 \\
\hline $\begin{array}{l}\text { Aishima et al. (2) } \\
\text { (38) }\end{array}$ & 2011 & Japan & $\begin{array}{l}\text { Peripheral } \\
\text { ICC }\end{array}$ & $69 / N R$ & NR & $\mathrm{HC}$ & $\begin{array}{l}\text { stained graded } \geq 2 \\
+\end{array}$ & $8(11.6 \%)$ & OS & $4.78(2.77-8.26)$ & Indirectly & 6 \\
\hline $\begin{array}{l}\text { Kawashima et al. } \\
\text { (36) }\end{array}$ & 2013 & Japan & EHBDC & $55 / 42$ & $\begin{array}{l}<65: 21 \\
\geq 65: 34\end{array}$ & $\mathrm{HC}$ & Stained cells $>1 \%$ & $40(72.7 \%)$ & OS & $8.51(1.18-64.8)$ & Directly & 7 \\
\hline Yuan et al. (35) & 2013 & China & $\mathrm{HCC}$ & $305 / 239$ & $\begin{array}{l}\text { Mean:55.09 } \\
\text { Range:15-88 }\end{array}$ & $\mathrm{HC}$ & stained cells $>1 \%$ & $173(56.7 \%)$ & OS & $1.91(1.22-2.99)$ & Directly & 6 \\
\hline Li et al. (33) & 2016 & China & GBC & $81 / 27$ & Mean:66.8 & $\mathrm{IHC}$ & stained cells $>5 \%$ & $50(61.7 \%)$ & OS & $1.40(0.84-2.34)$ & Indirectly & 6 \\
\hline $\begin{array}{l}\text { Nakayama et al. } \\
\text { (31) }\end{array}$ & 2019 & Japan & PC & $90 / 51$ & $\begin{array}{l}\text { Mean:66 } \\
\text { Range:36-85 }\end{array}$ & $\mathrm{HC}$ & NR & 45 (50\%) & OS, DFS & $1.92(0.99-3.72)$ & Indirectly & 6 \\
\hline
\end{tabular}


TABLE 2 | Summary of Newcastle-Ottawa quality assessment scale.

\begin{tabular}{|c|c|c|c|c|}
\hline \multicolumn{5}{|c|}{ Newcastle-Ottawa scale category } \\
\hline & Selection & Comparability & Outcome & Total \\
\hline Carneiro et al. (30) & 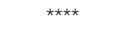 & * & $\star \star \star \star$ & 8 \\
\hline $\begin{array}{l}\text { Nakayama et al. } \\
\text { (31) }\end{array}$ & 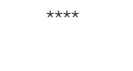 & / & 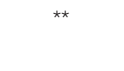 & 6 \\
\hline $\begin{array}{l}\text { Sarcognato et al. } \\
\text { (32) }\end{array}$ & 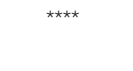 & / & $\star \star$ & 6 \\
\hline Wu et al. (11) & $\star \star \star \star ~$ & / & 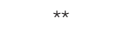 & 6 \\
\hline Shen et al. (23) & 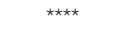 & * & $\star \star$ & 7 \\
\hline Li et al. (33) & $\star \star \star \star ~$ & / & 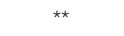 & 6 \\
\hline Dong et al. (13) & 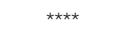 & * & $\star \star$ & 7 \\
\hline Sato et al. (34) & $\star \star \star \star ~$ & / & $\star \star$ & 6 \\
\hline Yuan et al. (35) & $\star \star \star \star ~$ & / & $\star \star$ & 6 \\
\hline Ge et al. (20) & $\star \star \star \star ~$ & / & $\star \star$ & 6 \\
\hline $\begin{array}{l}\text { Kawashima et al. } \\
\text { (36) }\end{array}$ & 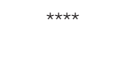 & * & $\star \star$ & 7 \\
\hline WANG, 2012 & 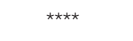 & * & $\star \star$ & 7 \\
\hline Tsai et al. (37) & $\star \star \star \star ~$ & / & $\star \star$ & 6 \\
\hline $\begin{array}{l}\text { Aishima et al. (1) } \\
\text { (38) }\end{array}$ & 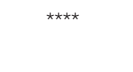 & / & ** & 6 \\
\hline $\begin{array}{l}\text { Aishima et al. (2) } \\
(38)\end{array}$ & 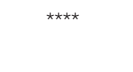 & / & $\star \star$ & 6 \\
\hline Zhao et al. (39) & 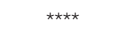 & / & $\star \star$ & 6 \\
\hline Jia et al. (40) & 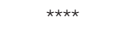 & / & $* \star$ & 6 \\
\hline
\end{tabular}

Score with an asterisk, *: one point; **: two points; ${ }^{* * *}$ : three points; ${ }^{* * *}$ : four points.
$(\mathrm{P}=0.319)$, detect method $(\mathrm{P}=0.773)$ and cut-off value $(\mathrm{P}=0.310)$ did not significantly contribute to the heterogeneity of OS (Table 3).

\section{Publication Bias}

Begg's funnel plots and Egger's test were used to estimate the potential publication bias. The results showed that there was no significant publication bias for OS according to Begg's test $(\mathrm{P}=0.650$, Figure 5A) and Egger's test $(\mathrm{P}=0.846$, Figure 5B $)$.

\section{DISCUSSION}

DSCs are a diverse group of tumors and have poor prognosis due to the advanced stage at the time of initial diagnosis. Despite that many achievements have been made in clinical and experimental studies, sensitive prognostic biomarkers are scarce, and reliable biomarkers are needed. Evidence has indicated that S100P overexpression is associated with poor prognosis in patients with some cancers $(16,17,20,23,24)$, but conflicting results have also been reported, indicating that S100P may enhance the chemosensitivity of GC (42). Therefore, it remains unknown whether S100P can serve as a biomarker to predict the prognosis of DSC patients. To evaluate the correlation between S100P expression and OS in DSC patients, 17 studies in 16

TABLE 3 | Subgroup analysis of the HRs of overall survival of patients with high S100P expression level.

\begin{tabular}{|c|c|c|c|c|c|c|c|c|}
\hline \multirow[t]{2}{*}{ Subgroup } & \multirow[t]{2}{*}{ No. of studies } & \multirow[t]{2}{*}{ No. of patients } & \multirow[t]{2}{*}{ HR $(95 \% \mathrm{Cl})$} & \multirow[t]{2}{*}{$P$ value } & \multicolumn{3}{|c|}{ Heterogeneity } & \multirow[t]{2}{*}{ Meta- regression, $p$ value } \\
\hline & & & & & $I^{2}(\%)$ & $P$ value & Model & \\
\hline Total & 17 & 1925 & $1.54(1.14,2.08)$ & 0.005 & 78.0 & $<0.001$ & random & \\
\hline Anatomic structure & & & & & & & & 0.758 \\
\hline Gastrointestinal tract & 7 & 1,000 & $1.09(0.66,1.81)$ & 0.727 & 83.1 & $<0.001$ & random & \\
\hline Non-gastrointestinal tract & 10 & 925 & $1.98(1.44,2.72)$ & $<0.001$ & 60.8 & 0.006 & random & \\
\hline Tumor type & & & & & & & & 0.925 \\
\hline Gastric cancer & 4 & 688 & $0.97(0.65,1.45)$ & 0.872 & 68.9 & 0.022 & random & \\
\hline Colon cancer + Colorectal cancer & 3 & 312 & $1.18(0.32,4.41)$ & 0.807 & 84.2 & 0.002 & random & \\
\hline Cholangiocarcinoma & 7 & 449 & $2.14(1.30,3.50)$ & 0.003 & 71.5 & 0.002 & random & \\
\hline Hepatocellular carcinoma & 1 & 305 & $1.91(1.22,2.99)$ & 0.005 & - & - & - & \\
\hline Gallbladder cancer & 1 & 81 & $1.40(0.84,2.34)$ & 0.198 & - & - & - & \\
\hline Pancreatic cancer & 1 & 90 & $1.92(0.99,3.72)$ & 0.053 & - & - & - & \\
\hline Sample size & & & & & & & & 0.456 \\
\hline$\geq 100$ & 6 & 1,137 & $1.57(1.06,2.33)$ & 0.024 & 79.1 & $<0.001$ & random & \\
\hline$<100$ & 11 & 788 & $1.50(0.93,2.43)$ & 0.098 & 79.5 & $<0.001$ & random & \\
\hline Study region & & & & & & & & 0.319 \\
\hline Europe & 2 & 1,546 & $1.31(0.87,1.98)$ & 0.190 & 56.5 & 0.130 & random & \\
\hline Asia & 15 & 379 & $1.57(1.10,2.26)$ & 0.014 & 79.5 & $<0.001$ & random & \\
\hline Detect method & & & & & & & & 0.773 \\
\hline $\mathrm{IHC}$ & 15 & 1,673 & $1.57(1.13,2.18)$ & 0.007 & 80.1 & $<0.001$ & random & \\
\hline RT-PCR & 1 & 156 & $1.80(1.02,3.19)$ & 0.044 & - & - & - & \\
\hline WB & 1 & 96 & $0.55(0.13,2.30)$ & 0.413 & - & - & - & \\
\hline Cut-off value & & & & & & & & 0.310 \\
\hline Stained grade $3+$ & 2 & 396 & $1.82(0.61,5.48)$ & 0.286 & 86.3 & 0.007 & random & \\
\hline Stained grade $2+$ & 5 & 417 & $2.04(1.06,3.93)$ & 0.032 & 86.6 & $<0.001$ & random & \\
\hline Stained cells $5 \%$ & 2 & 174 & $0.92(0.42,2.05)$ & 0.843 & 81.8 & 0.019 & random & \\
\hline Stained cells $1 \%$ & 3 & 472 & $1.84(1.27,2.67)$ & 0.001 & 24.6 & 0.266 & fixed & \\
\hline$N R$ & 3 & 342 & $1.62(0.99,2.65)$ & 0.054 & 21.5 & 0.280 & fixed & \\
\hline Others & 2 & 124 & $0.57(0.27,1.22)$ & 0.149 & 0.0 & 0.633 & fixed & \\
\hline
\end{tabular}

Cl, confidence interval; HR, hazard ratio; NR, no report; IHC, immunohistochemistry; RT-PCR, real-time polymerase chain reaction; WB, Western blotting; No., Number. 


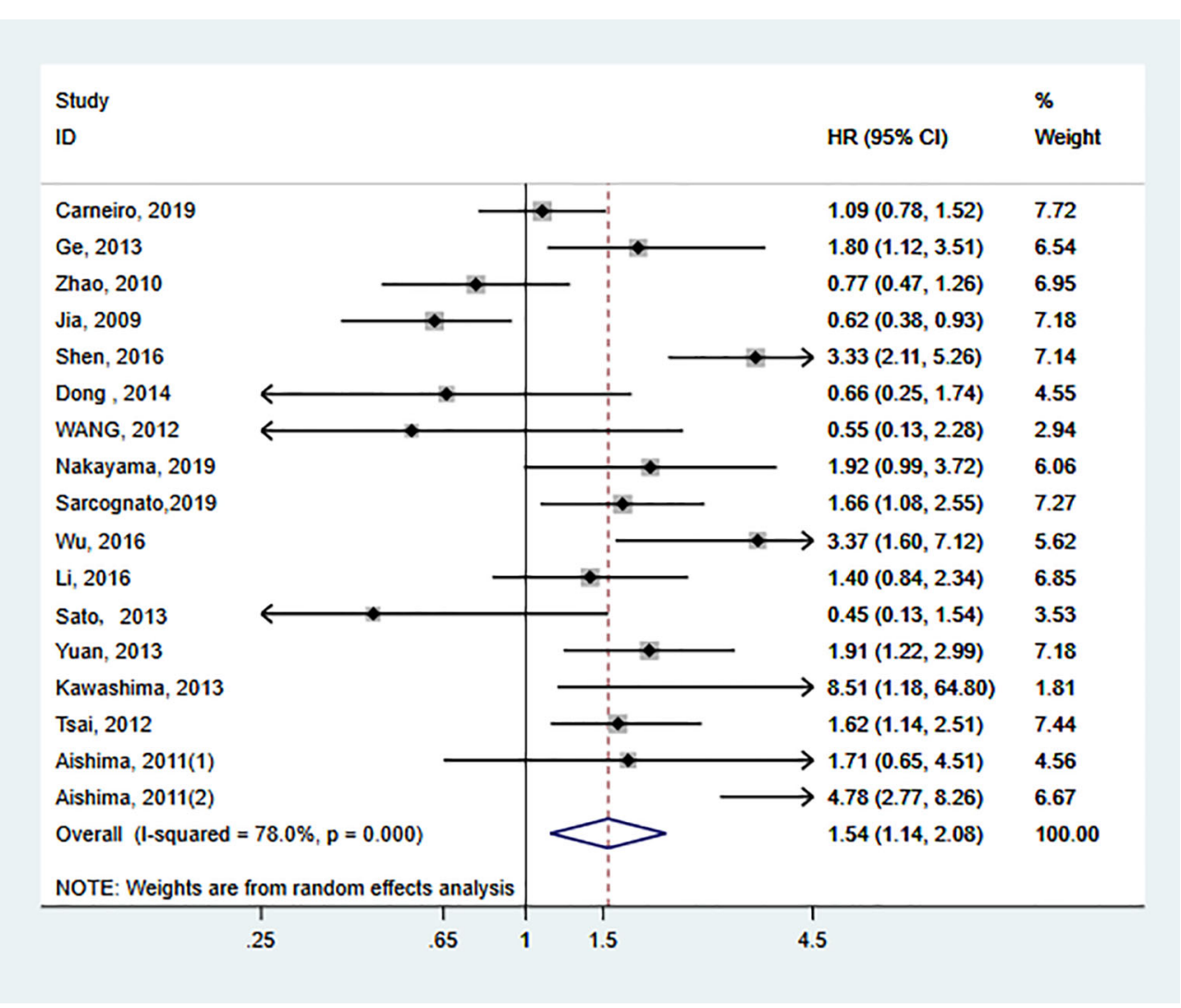

FIGURE 2 | Forest plot for the relationship between S100P expression and overall survival (OS).

publications with 1,925 patients were enrolled in the present meta-analysis $(11,13,20,23,27,30-40)$. In addition, we also explored the relationship between S100P expression and clinicopathological characteristics of DSCs.

Subgroup analyses by anatomic structure and tumor type were separately performed in DSC patients due to different TNM stage and prognosis among various tumor types. First, the prognostic value of S100P expression in all types of DSCs was evaluated. The results demonstrated that S100P overexpression was associated with poor OS in patients with DSCs, and higher predictive value of S100P was observed in non-gastrointestinal tract tumors compared to gastrointestinal tract tumors according to subgroup analysis. When subgroup analysis was performed by tumor type, the results revealed that $\mathrm{S} 100 \mathrm{P}$ overexpression was associated with poor OS in CCA and HCC. However, high S100P expression was not related to OS in gastric cancer, colorectal cancer, gallbladder cancer, and pancreatic cancer. Furthermore, when subgroup analysis was performed by sample size and study region, the pooled results were also significant in studies with large sample size and based on an Asian population but not in studies with small sample size and based on a European population. Sensitivity analysis, meta-regression analysis and publication bias tests suggested that these results were stable and credible.
Despite the robust results in the present meta-analysis, these findings should be interpreted with caution. First, the heterogeneity among the included studies was significant in our meta-analysis, even in the subgroup analyses. We analyzed that the significant heterogeneity may be caused by patient features, cancer types, ethnicity, literature quality, the detect methods and the cut-off values of S100P. So we performed a meta-regression analysis to identify the source of heterogeneity. However, none of these confounding factors completely explained the heterogeneity. Our meta-analysis showed that the prognostic value of high S100P expression varied with tumor types in DSC patients, which may be due to several mechanisms. First, S100P expression levels are varied in different tissues. Among the normal tissues, the highest S100P mRNA levels were detected in the placenta and esophagus. Moderate signals were detected in the stomach, duodenum, large intestine, prostate and leukocytes. At the protein level, the highest levels of S100P were detected in the placenta and stomach. Among the tumor tissues, however, S100P was most prevalent in gastric tumors (12). For those tissues with high S100P expression, S100P may play an important role in maintaining the normal status of cells. For those tissues with weak or no S100P expression with normal status, however, the elevated S100P expression level may have a relatively adverse 


\section{A sious}

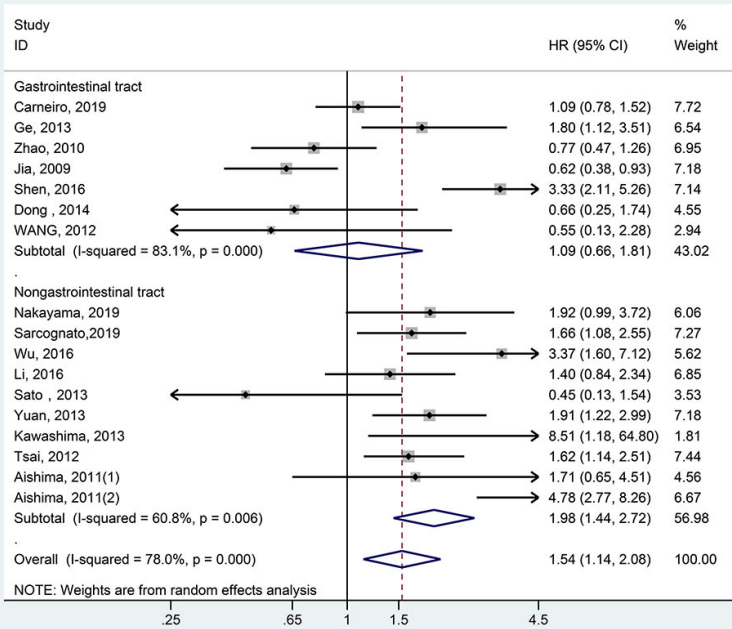

High S100P predicts better survival High S100P predicts poor survival

C

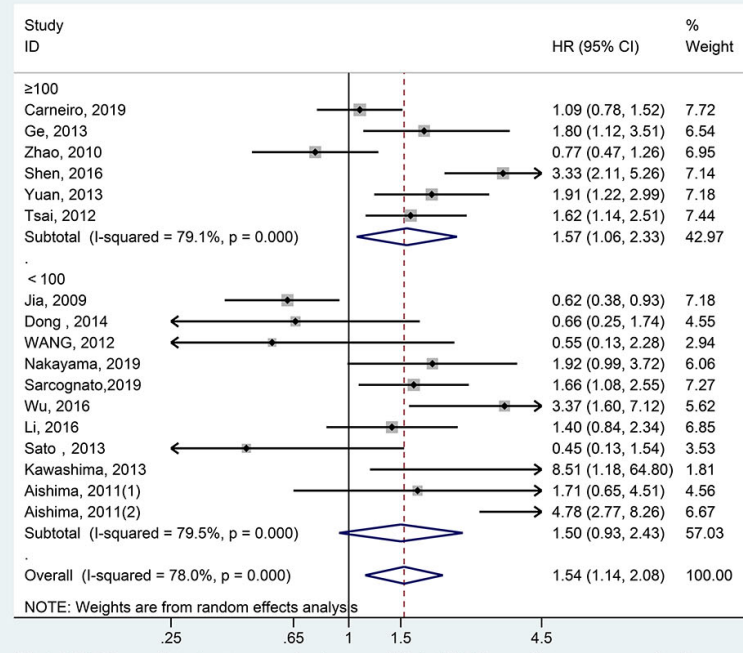

High S100P predicts better survival High S100P predicts poor survival
B

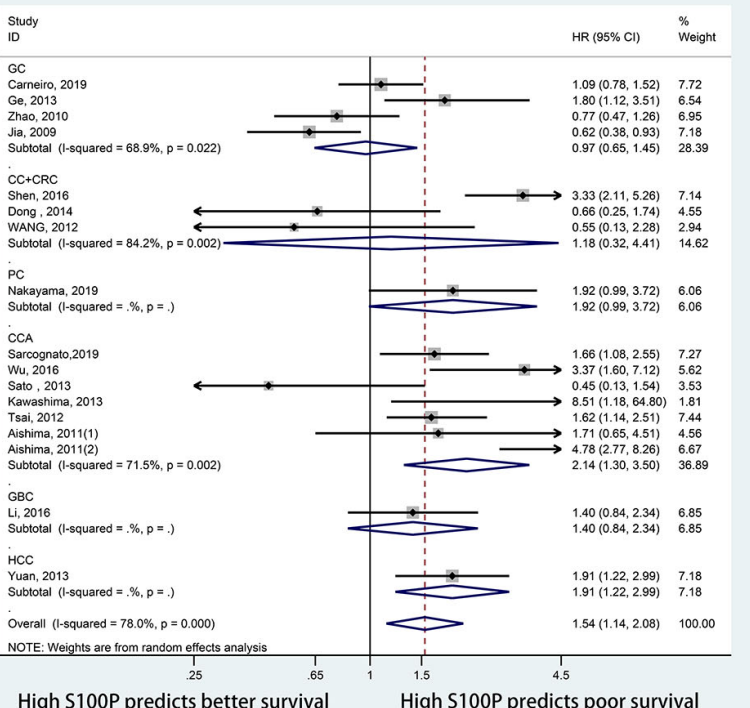

High S100P predicts better survival

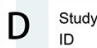

High S100P predicts poor survival

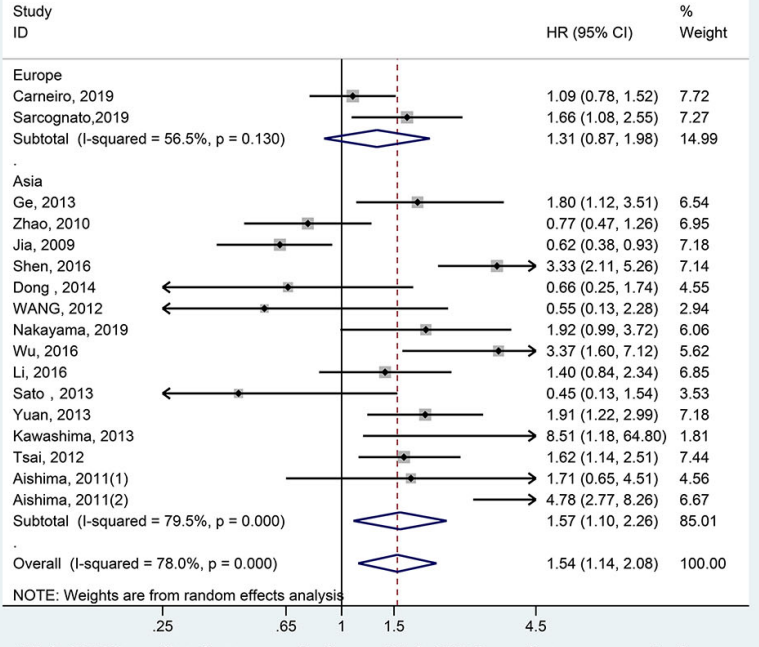

High S100P predicts better survival High S100P predicts poor survival

FIGURE 3 | Forest plots for the association between S100P expression and OS categorized by different subgroups: (A) Subgroup analysis of OS by anatomic structure; (B) Subgroup analysis of OS by cancer type; (C) Subgroup analysis of OS by sample size; (D) Subgroup analysis of OS by study region.

TABLE 4 | Pooled ORs for the relationship between S100P expression levels and clinicopathological parameters.

\begin{tabular}{|c|c|c|c|c|c|c|c|}
\hline Clinicopathological feature & $\begin{array}{l}\text { Number of } \\
\text { studies }\end{array}$ & $\begin{array}{l}\text { Number of } \\
\text { patients }\end{array}$ & OR (95\% Cl) & $\mathbf{P}$ & \multicolumn{3}{|c|}{ Heterogeneity } \\
\hline Gender (male vs female) & 9 & 1,301 & $0.91(0.63-1.32)$ & 0.617 & 51.4 & 0.036 & Random \\
\hline Lymph node metastasis (yes vs no) & 10 & 1,043 & $1.66(0.93-2.97)$ & 0.084 & 69.6 & 0.001 & Random \\
\hline $\begin{array}{l}\text { Distant metastasis } \\
\text { (yes vs no) }\end{array}$ & 4 & 405 & $3.58(1.04-12.36)$ & 0.044 & 81.3 & 0.001 & Random \\
\hline $\begin{array}{l}\text { Clinical stage } \\
\text { (III/IV vs I/II) }\end{array}$ & 7 & 820 & $2.03(1.03-4.01)$ & 0.041 & 77.0 & 0.001 & Random \\
\hline $\begin{array}{l}\text { Vascular invasion } \\
\text { (Present vs Absent) }\end{array}$ & 3 & 423 & $0.99(0.63-1.57)$ & 0.978 & 44.8 & 0.163 & Fixed \\
\hline Recurrence (yes vs no) & 3 & 506 & $1.66(1.15-2.38)$ & 0.007 & 48.3 & 0.144 & Fixed \\
\hline
\end{tabular}




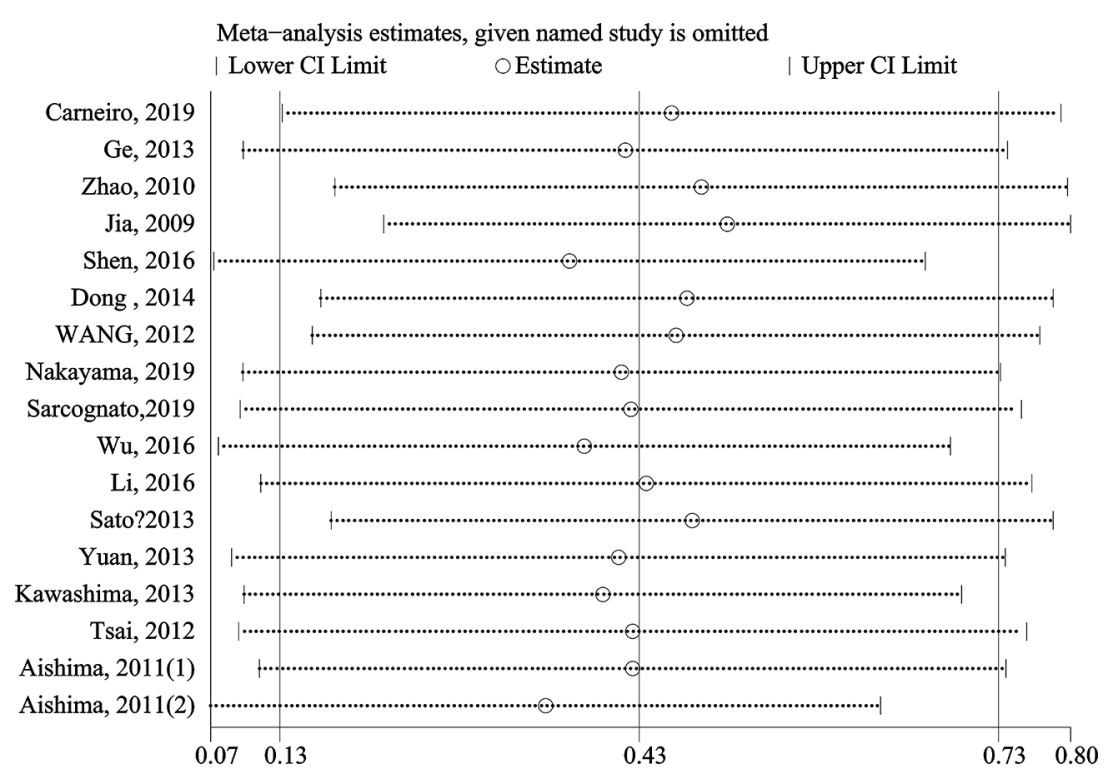

FIGURE 4 | Sensitivity analysis of S100P expression for OS.
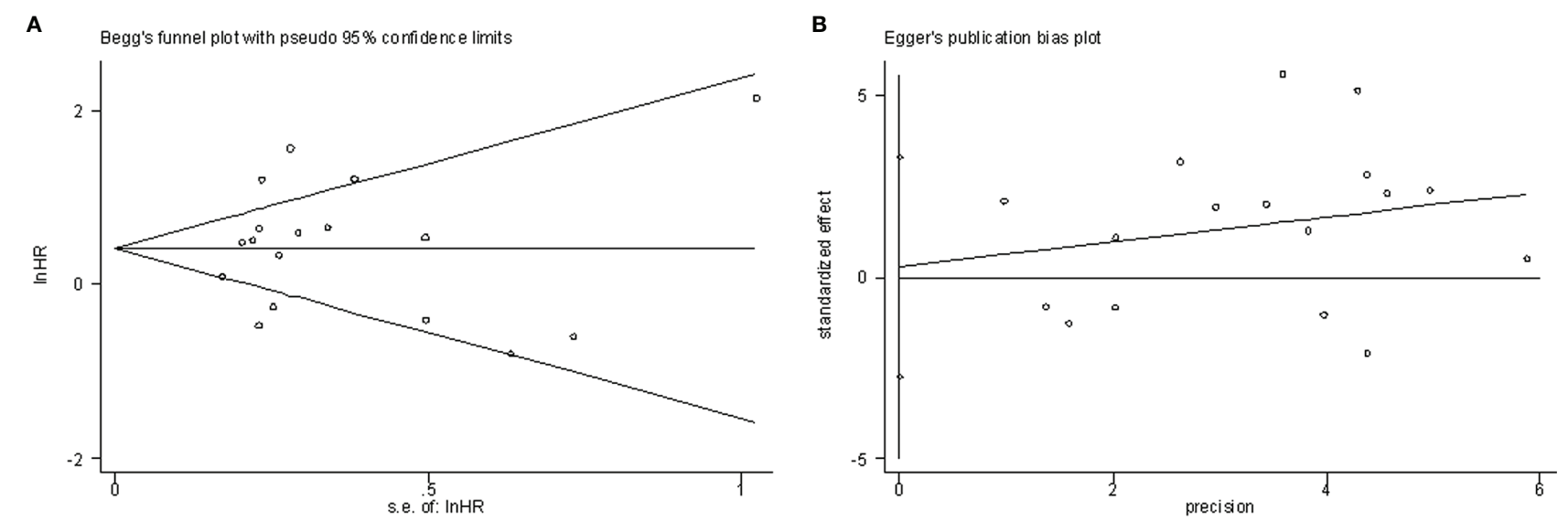

FIGURE 5 | Publication bias examination. (A) Begg's funnel plots assessing the publication bias for OS ( $\mathrm{p}=0.650)$; (B) Egger's test assessing the publication bias for OS $(p=0.846)$.

effect on the cells. Second, it has been reported that S100P is involved in various cellular functions $(5,7,8,43)$, indicating that it is a target protein or mediator protein for multiple signaling pathways $(33,44-46)$. These signaling pathways may play different roles in carcinogenesis in different tumors. Carneiro et al. (30) reported that S100P has a dual role in gastric cancer, acting as an oncogenic factor in the context of E-cadherin loss and as a tumor suppressor in a functional E-cadherin setting. Third, because only one article on hepatocellular carcinoma, gallbladder cancer and pancreatic cancer was included, the pooled HRs were weakly effective. Therefore, more studies are needed to investigate the functions of S100P in different cancers to explain the prognostic value of S100P.

Regarding the clinicopathological characteristics, our results indicated that higher expression levels of S100P indicated a greater possibility of distant metastasis, advance clinical stage and recurrence. Considering that the biology, pathology, clinical courses, and treatments vary among different types of DSCs, we also assessed the relationship between S100P expression and the clinicopathological characteristics in gastric cancer, colorectal cancer and cholangiocarcinoma. High S100P expression was not evaluated in hepatocellular carcinoma, gallbladder cancer, and 
pancreatic cancer due to limited data on the clinicopathological features. Furthermore, no significant correlation between S100P expression and clinicopathological characteristics in colorectal cancer was found, which may be due to the limited enrolled studies. S100P expression was significantly higher in females than in males in gastric cancer. Furthermore, increased S100P expression in cholangiocarcinoma was significantly associated with lymph node metastasis, which is consistent with the pooled results of OS in cholangiocarcinoma, suggesting that S100P overexpression may promote invasion and metastasis.

Our data suggested that S100P may be a potential reliable prognostic biomarker in some DSCs. The findings of the present study broadened and expanded the current understanding of S100P. However, there are several limitations that should be addressed. First, the number of 16 included publications for the topic of the digestive system seems small, especially when divided into six subgroups. And some report with small sample sizes. Due to the small number of included publications, our metaanalysis focus on the prognostic value of S100P in the digestive system which seems to be too broad. These may reduce the reliability of the results. But the sensitivity analysis, metaregression analysis and publication bias tests suggested that these results were stable and credible in our study. In the future, more studies with large sample sizes and low incidence tumors are needed. Second, some of the HRs were calculated from Kaplan-Meier survival curves rather than directly obtained from the primary data. Although the data of HRs are extracted by accepted methods, it would be better to obtain HRs directly from the literature. Third, patients enrolled in this meta-analysis were mostly from Asia, which may lead to selection bias. Fourth, although the meta-regression analysis showed that the detect method and cut-off value did not significantly contribute to the heterogeneity. But due to the small number of literatures in some subgroups, the actual situation may not be fully reflected. So we suspected that the detect methods and the cut-off values of S100P in studies included in meta-analysis were not consistent, which may relate to the heterogeneity of these studies. Unified detect methods and cut-off value should be used in the future study, and standardized conversion of cut-off values of different detection methods should be carried out, which is helpful to reduce heterogeneity in different studies and increase the reliability of the results.

\section{CONCLUSIONS}

In summary, our meta-analysis demonstrated that $\mathrm{S} 100 \mathrm{P}$ overexpression was associated with poor OS of DSC patients. The prognostic value of S100P expression was significant for the OS of patients with cholangiocarcinoma and hepatocellular carcinoma but not for patients with gastric cancer, colorectal cancer, gallbladder cancer, and pancreatic cancer. When stratified by anatomic structure, S100P overexpression was associated with poor prognosis in non-gastrointestinal tract cancers but not in gastrointestinal tract cancers. Furthermore, significant correlation was observed between S100P expression and some phenotypes of tumor aggressiveness, such as distant metastasis, advanced clinical stage, and recurrence. These results indicated that S100P may be an effective factor of poor prognosis in some digestive system cancers, especially in non-gastrointestinal tract cancers. Nevertheless, as the study had several limitations, further large-scale, well-designed studies are needed to confirm our results.

\section{DATA AVAILABILITY STATEMENT}

The raw data supporting the conclusions of this article will be made available by the authors, without undue reservation.

\section{AUTHOR CONTRIBUTIONS}

B-XL, C-TT, and X-JD were responsible for study design. B-XL and C-TT were responsible for literature search. B-XL and C-TT were responsible for data extraction. X-JD, LZ, and FC were responsible for data analysis. $\mathrm{B}-\mathrm{XL}$ and $\mathrm{C}-\mathrm{TT}$ were responsible for drafting the manuscript. $\mathrm{CZ}$ and $\mathrm{YC}$ approved the final version of the manuscript. All authors contributed to the article and approved the submitted version.

\section{FUNDING}

This study was supported by grants from the Key Foundation of Jiangxi Provincial Department of Science and Technology (grant No. 20202ACBL206018), the Foundation of Jiangxi Provincial Department of Science and Technology (grant No. 20202BAB206051), the Foundation of Jiangxi Provincial Department of Science and Technology (grant No. 20201ZDG02007), the National Natural Science Foundation of China (grant No. 81701678), the Science and Technology Foundation of Jiangxi Provincial Health Commission (grant No. 20191098), and the China Postdoctoral Science Foundation (grant No, 2020M670052). Sanming Project of Medicine in Shenzhen (grant No, SZSM201812052).

\section{SUPPLEMENTARY MATERIAL}

The Supplementary Material for this article can be found online at: https://www.frontiersin.org/articles/10.3389/fonc.2021. 593728/full\#supplementary-material

Supplementary Figure 1 | Forest plots for the association between S100P expression and OS categorized by detect methods.

Supplementary Figure 2 | Forest plots for the association between S100P expression and OS categorized by cut-off value. 


\section{REFERENCES}

1. Siegel RL, Miller KD, Jemal A. Cancer statistics, 2020. CA Cancer J Clin (2020) 70(1):7-30. doi: 10.3322/caac.21590

2. Ng L, Poon RT, Pang R. Biomarkers for predicting future metastasis of human gastrointestinal tumors. Cell Mol Life Sci (2013) 70(19):3631-56. doi: 10.1007/ s00018-013-1266-8

3. Grandhi MS, Kim AK, Ronnekleiv-Kelly SM, Kamel IR, Ghasebeh MA, Pawlik TM. Hepatocellular carcinoma: From diagnosis to treatment. Surg Oncol (2016) 25(2):74-85. doi: 10.1016/j.suronc.2016.03.002

4. Zhao QT, Yang Y, Xu S, Zhang XP, Wang HE, Zhang H, et al. Prognostic role of neutrophil to lymphocyte ratio in lung cancers: a meta-analysis including 7,054 patients. Onco Targets Ther (2015) 8:2731-8. doi: 10.2147/OTT.S90875

5. Becker T, Gerke V, Kube E, Weber K. S100P, a novel Ca(2+)-binding protein from human placenta. cDNA cloning, recombinant protein expression and Ca2+ binding properties. Eur J Biochem (1992) 207(2):541-7. doi: 10.1111/ j.1432-1033.1992.tb17080.x

6. Emoto Y, Kobayashi R, Akatsuka H, Hidaka H. Purification and characterization of a new member of the S-100 protein family from human placenta. Biochem Biophys Res Commun (1992) 182(3):1246-53. doi: 10.1016/ 0006-291X(92)91865-N

7. Tothova V, Gibadulinova A. S100P, a peculiar member of S100 family of calcium-binding proteins implicated in cancer. Acta Virol (2013) 57(2):23846. doi: 10.4149/av_2013_02_238

8. Prica F, Radon T, Cheng Y, Crnogorac-Jurcevic T. The life and works of S100P - from conception to cancer. Am J Cancer Res (2016) 6(2):562-76.

9. Zuo Z, Zhang P, Lin F, Shang W, Bi R, Lu F, et al. Interplay between Trx-1 and S100P promotes colorectal cancer cell epithelial-mesenchymal transition by up-regulating S100A4 through AKT activation. J Cell Mol Med (2018) 22 (4):2430-41. doi: $10.1111 / \mathrm{jcmm} .13541$

10. Du M, Wang G, Ismail TM, Gross S, Fernig DG, Barraclough R, et al. S100P dissociates myosin IIA filaments and focal adhesion sites to reduce cell adhesion and enhance cell migration. J Biol Chem (2012) 287(19):15330-44. doi: 10.1074/jbc.M112.349787

11. Wu Z, Boonmars T, Nagano I, Boonjaraspinyo S, Srinontong P, Ratasuwan P, et al. Significance of $\mathrm{S} 100 \mathrm{P}$ as a biomarker in diagnosis, prognosis and therapy of opisthorchiasis-associated cholangiocarcinoma. Int J Cancer (2016) 138 (2):396-408. doi: 10.1002/ijc.29721

12. Parkkila S, Pan PW, Ward A, Gibadulinova A, Oveckova I, Pastorekova S, et al. The calcium-binding protein S100P in normal and malignant human tissues. BMC Clin Pathol (2008) 8:2. doi: 10.1186/1472-6890-8-2

13. Dong L, Wang F, Yin X, Chen L, Li G, Lin F, et al. Overexpression of S100P promotes colorectal cancer metastasis and decreases chemosensitivity to 5-FU in vitro. Mol Cell Biochem (2014) 389(1):257-64. doi: 10.1007/s11010-013-1947-5

14. Birkenkamp-Demtroder K, Olesen SH, Sørensen FB, Laurberg S, Laiho P, Aaltonen LA, et al. Differential gene expression in colon cancer of the caecum versus the sigmoid and rectosigmoid. Gut (2005) 54(3):374-84. doi: 10.1136/ gut.2003.036848

15. Ni X, Chen J, Lu F, Yuan Z, Xu X, Hu Z, et al. Anti-Cancer Effect of alphaSolanine by Down-Regulating S100P Expression in Colorectal Cancer Cells. Recent Pat Anticancer Drug Discov (2018) 13(2):240-7. doi: 10.2174/ 1574892813666180329163945

16. Hsu YL, Hung JY, Liang YY, Lin YS, Tsai MJ, Chou SH, et al. S100P interacts with integrin alpha7 and increases cancer cell migration and invasion in lung cancer. Oncotarget (2015) 6(30):29585-98. doi: 10.18632/oncotarget.4987

17. Arumugam T, Simeone DM, Van Golen K, Logsdon CD. S100P promotes pancreatic cancer growth, survival, and invasion. Clin Cancer Res (2005) 11 (15):5356-64. doi: 10.1158/1078-0432.CCR-05-0092

18. Kikuchi K, McNamara KM, Miki Y, Iwabuchi E, Kanai A, Miyashita M, et al. S100P and Ezrin promote trans-endothelial migration of triple negative breast cancer cells. Cell Oncol (Dordr) (2019) 42(1):67-80. doi: 10.1007/s13402-0180408-2

19. Wang X, Tian T, Li X, Zhao M, Lou Y, Qian J, et al. High expression of S100P is associated with unfavorable prognosis and tumor progression in patients with epithelial ovarian cancer. Am J Cancer Res (2015) 5(8):2409-21.

20. Ge F, Wang C, Wang W, Wu B. S100P predicts prognosis and drug resistance in gastric cancer. Int J Biol Markers (2013) 28(4):e387-92. doi: 10.5301/ JBM.5000034
21. Arumugam T, Simeone DM, Schmidt AM, Logsdon CD. S100P stimulates cell proliferation and survival via receptor for activated glycation end products (RAGE). J Biol Chem (2004) 279(7):5059-65. doi: 10.1074/jbc.M310124200

22. Austermann J, Nazmi AR, Müller-Tidow C, Gerke V. Characterization of the $\mathrm{Ca} 2+$-regulated ezrin-S100P interaction and its role in tumor cell migration. J Biol Chem (2008) 283(43):29331-40. doi: 10.1074/jbc.M806145200

23. Shen ZY, Fang Y, Zhen L, Zhu XJ, Chen H, Liu H, et al. Analysis of the predictive efficiency of $\mathrm{S} 100 \mathrm{P}$ on adverse prognosis and the pathogenesis of S100P-mediated invasion and metastasis of colon adenocarcinoma. Cancer Genet (2016) 209(4):143-53. doi: 10.1016/j.cancergen.2016.02.002

24. Guerreiro DSI, Hu YF, Russo IH, Ao X, Salicioni AM, Yang X, et al. S100P calcium-binding protein overexpression is associated with immortalization of human breast epithelial cells in vitro and early stages of breast cancer development in vivo. Int J Oncol (2000) 16(2):231-40. doi: 10.3892/ijo.16.2.231

25. Barry S, Chelala C, Lines K, Sunamura M, Wang A, Marelli-Berg FM, et al. $\mathrm{S} 100 \mathrm{P}$ is a metastasis-associated gene that facilitates transendothelial migration of pancreatic cancer cells. Clin Exp Metastasis (2013) 30(3):25164. doi: 10.1007/s10585-012-9532-y

26. Dakhel S, Padilla L, Adan J, Masa M, Martinez JM, Roque L, et al. S100P antibody-mediated therapy as a new promising strategy for the treatment of pancreatic cancer. Oncogenesis (2014) 3:e92. doi: 10.1038/oncsis.2014.7

27. Wang Q, Zhang YN, Lin GL, Qiu HZ, Wu B, Wu HY, et al. S100P, a potential novel prognostic marker in colorectal cancer. Oncol Rep (2012) 28(1):303-10. doi: 10.3892/or.2012.1794

28. Moher D, Liberati A, Tetzlaff J, Altman DG, PRISMA Group. Preferred reporting items for systematic reviews and meta-analyses: the PRISMA statement. BMJ (2009) 339:b2535. doi: 10.1136/bmj.b2535

29. Stang A. Critical evaluation of the Newcastle-Ottawa scale for the assessment of the quality of nonrandomized studies in meta-analyses. Eur J Epidemiol (2010) 25(9):603-5. doi: 10.1007/s10654-010-9491-Z

30. Carneiro P, Moreira AM, Figueiredo J, Barros R, Oliveira P, Fernandes MS, et al. S100P is a molecular determinant of E-cadherin function in gastric cancer. Cell Commun Signal (2019) 17(1):155. doi: 10.1186/s12964-019-0465-9

31. Nakayama H, Ohuchida K, Yonenaga A, Sagara A, Ando Y, Kibe S, et al. S100P regulates the collective invasion of pancreatic cancer cells into the lymphatic endothelial monolayer. Int J Oncol (2019) 55(1):211-22. doi: 10.3892/ijo.2019.4812

32. Sarcognato S, Gringeri E, Fassan M, Di Giunta M, Maffeis V, Guzzardo V, et al. Prognostic role of BAP-1 and PBRM-1 expression in intrahepatic cholangiocarcinoma. Virchows Arch (2019) 474(1):29-37. doi: 10.1007/ s00428-018-2478-y

33. Li Z, Chen Y, Wang X, Zhang H, Zhang Y, Gao Y, et al. LASP-1 induces proliferation, metastasis and cell cycle arrest at the G2/M phase in gallbladder cancer by down-regulating S100P via the PI3K/AKT pathway. Cancer Lett (2016) 372(2):239-50. doi: 10.1016/j.canlet.2016.01.008

34. Sato Y, Harada K, Sasaki M, Nakanuma Y. Clinicopathological significance of S100 protein expression in cholangiocarcinoma. J Gastroenterol Hepatol (2013) 28(8):1422-9. doi: 10.1111/jgh.12247

35. Yuan RH, Chang KT, Chen YL, Hsu HC, Lee PH, Lai PL, et al. S100P expression is a novel prognostic factor in hepatocellular carcinoma and predicts survival in patients with high tumor stage or early recurrent tumors. PLoS One (2013) 8(6):e65501. doi: 10.1371/journal.pone.0065501

36. Kawashima H, Itoh A, Ohno E, Miyahara R, Ohmiya N, Tanaka T, et al. Diagnostic and prognostic value of immunohistochemical expression of S100P and IMP3 in transpapillary biliary forceps biopsy samples of extrahepatic bile duct carcinoma. J Hepato Biliary Pancreatic Sci (2013) 20 (4):441-7. doi: 10.1007/s00534-012-0581-z

37. Tsai JH, Huang WC, Kuo KT, Yuan RH, Chen YL, Jeng YM. S100P immunostaining identifies a subset of peripheral-type intrahepatic cholangiocarcinomas with morphological and molecular features similar to those of perihilar and extrahepatic cholangiocarcinomas. Histopathology (2012) 61(6):1106-16. doi: 10.1111/j.1365-2559.2012.04316.x

38. Aishima S, Fujita N, Mano Y, Kubo Y, Tanaka Y, Taketomi A, et al. Different roles of S100P Overexpression in Intrahepatic Cholangiocarcinoma. Am J Surg Pathol (2011) 35(4):590-8. doi: 10.1097/PAS.0b013e31820ffdf1

39. Zhao XM, Bai ZG, Ma XM, Zhang ZT, Shi XY. [Impact of S100P expression on clinical outcomes of gastric cancer patients with adjuvant chemotherapy of oxaliplatin and its mechanisms]. Zhonghua Wai Ke Za Zhi (2010) 48(13):1004-8. 
40. Jia SQ, Niu ZJ, Zhang LH, Zhong XY, Shi T, Du H, et al. Identification of prognosis-related proteins in advanced gastric cancer by mass spectrometrybased comparative proteomics. J Cancer Res Clin Oncol (2009) 135(3):403-11. doi: 10.1007/s00432-008-0474-3

41. Blechacz B. Cholangiocarcinoma: Current Knowledge and New Developments. Gut Liver (2017) 11(1):13-26. doi: 10.5009/gnl15568

42. Zhao X, Bai Z, Wu P, Zhang Z. S100P enhances the chemosensitivity of human gastric cancer cell lines. Cancer Biomark (2013) 13(1):1-10. doi: 10.3233/CBM-130330

43. Jiang H, Hu H, Tong X, Jiang Q, Zhu H, Zhang S. Calcium-binding protein S100P and cancer: mechanisms and clinical relevance. J Cancer Res Clin Oncol (2012) 138(1):1-9. doi: 10.1007/s00432-011-1062-5

44. Shen Z, Deng H, Fang Y, Zhu X, Ye GT, Yan L. Identification of the interplay between SOX9 and S100P in the metastasis and invasion of colon carcinoma. Oncotarget (2015) 6(24):20672-84. doi: 10.18632/oncotarget.3967

45. Mercado-Pimentel ME, Onyeagucha BC, Li Q, Pimentel AC, Jandova J, Nelson MA. The S100P/RAGE signaling pathway regulates expression of
microRNA-21 in colon cancer cells. FEBS Lett (2015) 589(18):2388-93. doi: 10.1016/j.febslet.2015.07.010

46. Zhang Q, Hu H, Shi X, Tang W. Knockdown of S100P by lentiviral-mediated RNAi promotes apoptosis and suppresses the colony-formation ability of gastric cancer cells. Oncol Rep (2014) 31(5):2344-50. doi: 10.3892/ or.2014.3104

Conflict of Interest: The authors declare that the research was conducted in the absence of any commercial or financial relationships that could be construed as a potential conflict of interest.

Copyright @ 2021 Liu, Tang, Dai, Zeng, Cheng, Chen and Zeng. This is an open-access article distributed under the terms of the Creative Commons Attribution License (CC BY). The use, distribution or reproduction in other forums is permitted, provided the original author(s) and the copyright owner(s) are credited and that the original publication in this journal is cited, in accordance with accepted academic practice. No use, distribution or reproduction is permitted which does not comply with these terms. 